\title{
Additive manufacturing of reflective optics: evaluating finishing methods
}

\section{G. Leuteritz, R. Lachmayer}

G. Leuteritz, R. Lachmayer, "Additive manufacturing of reflective optics: evaluating finishing methods," Proc. SPIE 10523, Laser 3D Manufacturing V, 105230N (26 February 2018); doi: 10.1117/12.2289998

SPIE. Event: SPIE LASE, 2018, San Francisco, California, United States 


\title{
Additive Manufacturing of Reflective Optics: Evaluating Finishing Methods
}

\author{
G. Leuteritz*a $*^{\mathrm{a}}$, R. Lachmayer ${ }^{\mathrm{a}}$ \\ ${ }^{\mathrm{a}}$ Gottfried Wilhelm Leibniz University, Institute of Product Development, Welfengarten 1A, 30167 \\ Hannover, Lower Saxony, Germany
}

\begin{abstract}
Individually shaped light distributions become more and more important in lighting technologies and thus the importance of additively manufactured reflectors increases significantly. The vast field of applications ranges from automotive lighting to medical imaging and bolsters the statement. However, the surfaces of additively manufactured reflectors suffer from insufficient optical properties even when manufactured using optimized process parameters for the Selective Laser Melting (SLM) process. Therefore post-process treatments of reflectors are necessary in order to further enhance their optical quality.

This work concentrates on the effectiveness of post-process procedures for reflective optics. Based on already optimized aluminum reflectors, which are manufactured with a SLM machine, the parts are differently machined after the SLM process. Selected finishing methods like laser polishing, sputtering or sand blasting are applied and their effects quantified and compared.

The post-process procedures are investigated on their impact on surface roughness and reflectance as well as geometrical precision. For each finishing method a demonstrator will be created and compared to a fully milled sample and among themselves. Ultimately, guidelines are developed in order to figure out the optimal treatment of additively manufactured reflectors regarding their optical and geometrical properties. Simulations of the light distributions will be validated with the developed demonstrators.
\end{abstract}

Keywords: Additive Manufacturing, finishing methods, reflective optics

\section{INTRODUCTION}

Additive Manufacturing sums up several manufacturing processes that evolved to be the most impressive and forwardthinking processes in the present days. Technologies like Selective Laser Melting or Stereolithography are established in a broad range of industries and applications such as bioengineering or optics ${ }^{1,2}$. Based on the layer-by-layer building process an unparalleled freedom of design has arisen, which leads to new opportunities in designing parts and the possibility to integrate several functions within one part. What started with the manufacturing of functional prototypes has been developed into a technique which is able to directly manufacture complicated and highly integrated parts and even systems ${ }^{3,4}$.

However, the manufacturing processes are not flawless and it often takes further effort to gain the desired properties of the part. Surfaces can be too rough, support structures have to be removed or a thermal treatment is necessary in order to reduce internal stresses caused during the manufacturing process. These finishing methods are essential for the product quality and cannot be neglected. With that in mind the term direct manufacturing - the manufacturing of components with net shape geometry and desired properties within one system - has to be treated carefully when it is used in relation to additive manufacturing. ${ }^{5,6}$

*leuteritz@ipeg.uni-hannover.de; phone +49 511762 4338; fax +49 511762 4506; www.ipeg.uni-hannover.de 
To implement direct manufacturing many investigations focus on the integration of more than one manufacturing technique into one system. These hybrid systems often combine the advantages of an additive manufacturing process and an abrasive process such as milling ${ }^{7,8,9}$. Complex geometries can be built and at the same time the geometrical tolerances are kept to a minimum and the surfaces can be treated further. For example, additional heating devices are used for reducing stresses.

As the description suggests the designated function of the manufactured parts is of mechanical nature. Those parts endure dynamic or static loads and stresses.

There is also research about the fabrication of optical components. Those are mainly waveguides and freeform lenses that are manufactured by stereolithographic systems. Transparent polymer structures are formed using UV light in order to force a polymerization. Depending on the process, the removal of support structures and remaining material on the component are necessary. Furthermore the components have to be exposed to UV light after the manufacturing process for complete polymerization to ensure the optical properties. The most commercially available systems are equipped with such post-processing techniques, but freeform lenses still need to be treated mechanically due to their surface roughness in order to suffice for high precision applications. ${ }^{10,11}$

A field of application that has not yet been properly addressed is the manufacturing of reflective optics. Reflectors are mostly die casted and coated afterwards. Nevertheless the need for individual light distributions and hence individually designed reflectors cannot be efficiently compensated using conventional manufacturing techniques. And as already described the surface roughness and thereby the optical quality of the reflector will be insufficient for precision applications.

This works purpose is to describe the different possibilities of post-process treatment for additively manufactured reflector samples made of AlSi10Mg. The samples are fabricated with the Selective Laser Melting process, which has been adapted in order to achieve surface roughness as low as possible. The post-processes are categorized and their influences on the surface properties are shown. This includes the surface roughness and also the reflectivity of the samples. According to the influence and the treatment effort a ranking is set up to evaluate the different techniques. In conclusion a suggestion for integrating post-process techniques into an additive manufacturing process is offered in order to promote the direct manufacturing of reflective optics.

\section{ESTABLISHED FINISHING METHODS}

This section describes the state of the art post-process techniques and an approach for categorizing them. The techniques, which are described here, are specifically for enhancing surface qualities since these properties are decisive for the functionality of the manufactured reflectors.

\subsection{Categorization of post-process techniques}

There are at least two approaches on how to categorize post-process techniques. The first one is based the physical principle that is used for modifying the components. In this case the first level distinguishes between optical, chemical, thermal and mechanical post-process treatments. Possible examples are milling (mechanical), laser polishing (optical), stress-relief annealing (thermal) and plasma polishing (chemical). ${ }^{12,13}$

Another approach, which will be considered for this work, is the differentiation between the processes that happen on the components surface. Therefore the categories are transformative, abrasive and additive techniques. Typical examples are laser polishing (transformative), milling (abrasive) and coating (additive). A schematic of this categorization can be seen in figure 1.

The schematic demonstrates the imbalance of possibilities for each category of finishing methods. Abrasive techniques are widely spread for a broad range of applications. Furthermore some of these techniques are not only used as finishing techniques but manufacturing techniques as well. Milling for example has been established for both areas and is used for the fabrication of metal and polymer parts as well as enhancement of additively manufactured optics. ${ }^{14}$ 


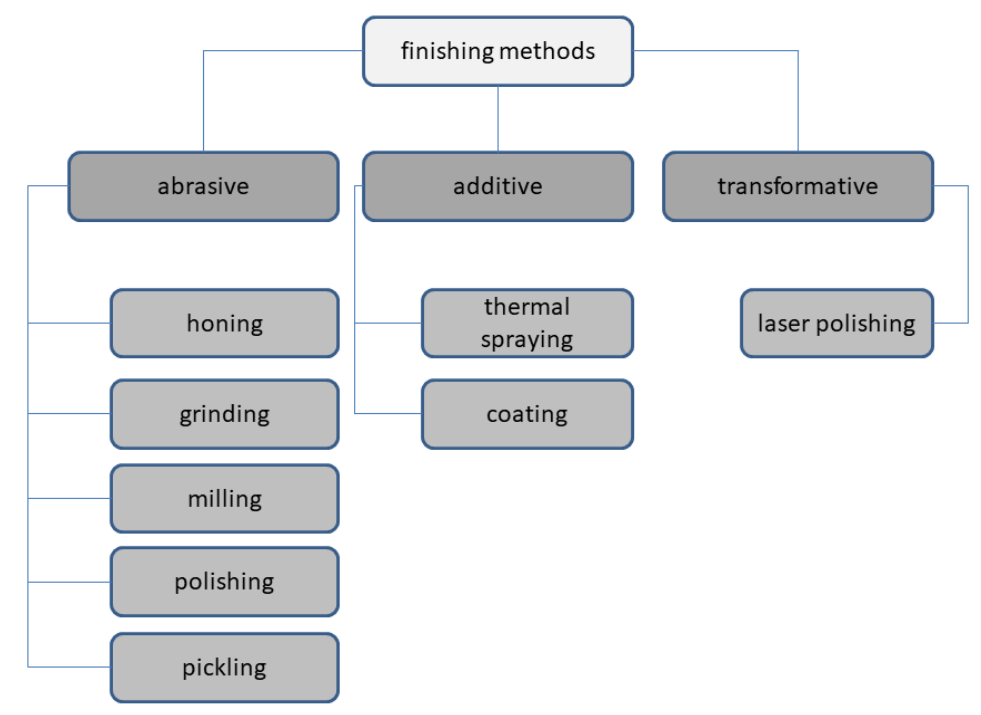

Figure 1: categorization of finishing methods with selected examples

However, with this categorization the question remains, which one of these categories and which technique in particular are best suited for enhancing reflective optics and therefore should be integrated in Additive Manufacturing machines.

\subsection{Hybrid manufacturing}

Hybrid machines that combine the main manufacturing process with an additional finishing setup within one system are already vastly introduced to scientific as well as industrial purposes ${ }^{9}$. The variety of different machines allows yet another categorization, which is shown in figure 2.

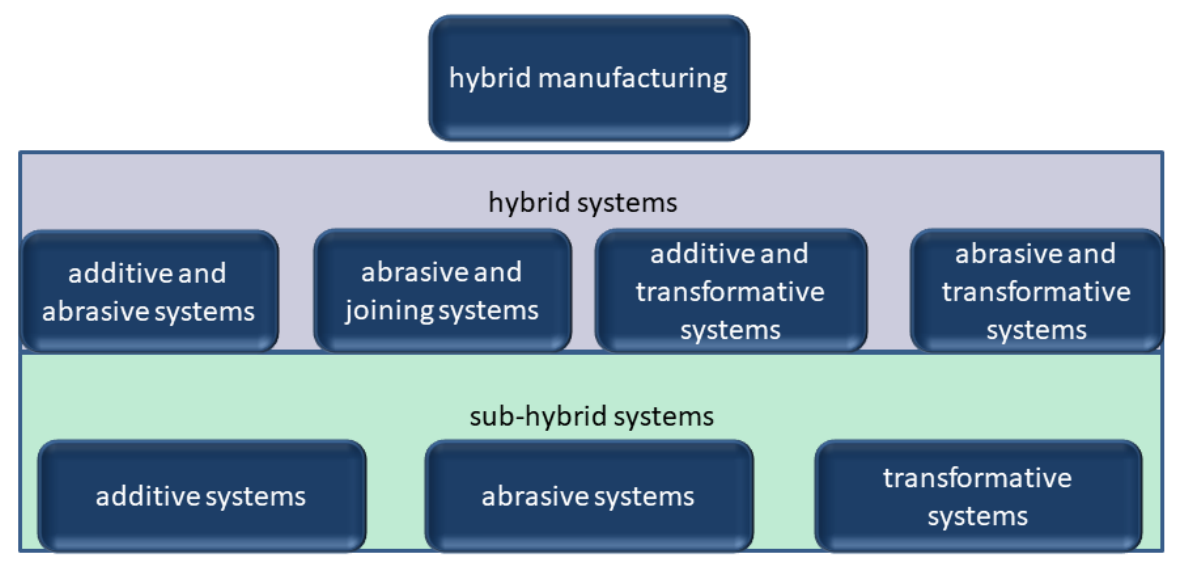

Figure 2: categorization of hybrid manufacturing systems according to Zhu et al. ${ }^{9}$

Within this differentiation the combination of two processes from different manufacturing categories is called hybrid system and the combination of two processes from the same manufacturing category is a sub-hybrid system. For additive manufacturing most hybrid systems are equipped with a milling system for finishing tasks. These systems, although using the processes, can be realized very differently, depending on the AM process that is used for manufacturing. Two 
industrial setups, one from DMG Mori and one from Additive Industries, present hybrid manufacturing, which can be seen in figure 3.
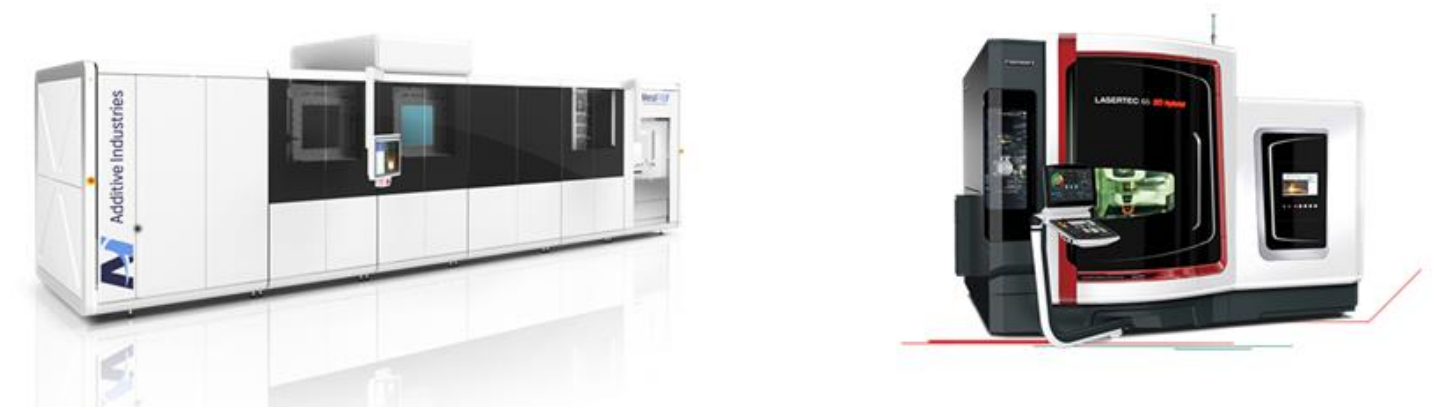

Figure 3: Hybrid system of Additive Industries using several chambers for processing (left) and DMG Mori with postprocessing within the same building chamber (right)

Since the setup of Additive Industries uses a Selective Laser Melting process the finishing steps cannot be performed within the same chamber. Therefore the fabricated part is transferred to several post-process chambers that include thermal treatments and surface enhancements. One of the systems introduced by DMG Mori however uses a nozzlebased approach which allows the local application of metal powder. This offers the opportunity to integrate a milling device within the same building chamber, since there is no powder bed which disturbs the milling process. Additionally the whole system is modified with a 5-axis movement, which allows high precision manufacturing regardless of the building angle.

Although these and many other machines offer hybrid solutions for manufacturing, limitations occur in a way that the fabricated parts are mostly used for mechanical purposes. Therefore the question remains, which post-process technique fits best for the fabrication of reflective optics and whether a single post-process can be enough to ensure a sufficient surface quality. The last objective of this investigation addresses the suitability of integrating a technique in Additive Manufacturing systems for hybrid manufacturing in order to suggest a system for direct manufacturing of reflective optics. Since the majority of post-process techniques are of this category the following research concentrates on mechanical post-processing.

\section{METHODS AND MATERIALS}

In this section the investigated sample geometry and measurement methodology are introduced. Furthermore the postprocessing sequence is demonstrated.

\subsection{Sample properties}

The geometry of the samples can be seen in figure 4. The samples are made of AlSi10Mg. Aluminum is often used for the manufacturing of reflective optics because of its low density and high reflectivity within the visible spectrum. An alternative to aluminum is magnesium. The sole purpose of these samples is to investigate their surface properties for different building angles. Since the reflective surface of a reflector does not exceed the surface of a half sphere, the maximum surface angle for the samples is set to $90^{\circ}$. The behavior of the surface properties is expected to be rotationally symmetric to the $\mathrm{z}$-axis (building direction), which reduces the required positions of the sample in the building space to one. 


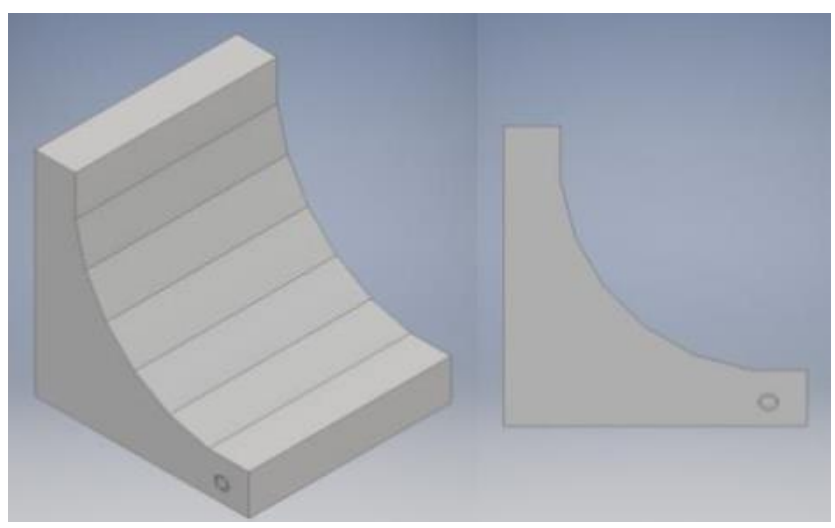

Figure 4: Samples for surface investigations; the facets are tilted within surface angles from $0^{\circ}$ to $90^{\circ}$

The reflective surfaces will always be oriented as up-skin surfaces in order to avoid support structures. Down-skin surfaces can imply an additional post-processing effort and further limit the suitable techniques to abrasive processes.

\subsection{Post-Processing and measurement}

The mechanical post-processing is structured as depicted in figure 5 .

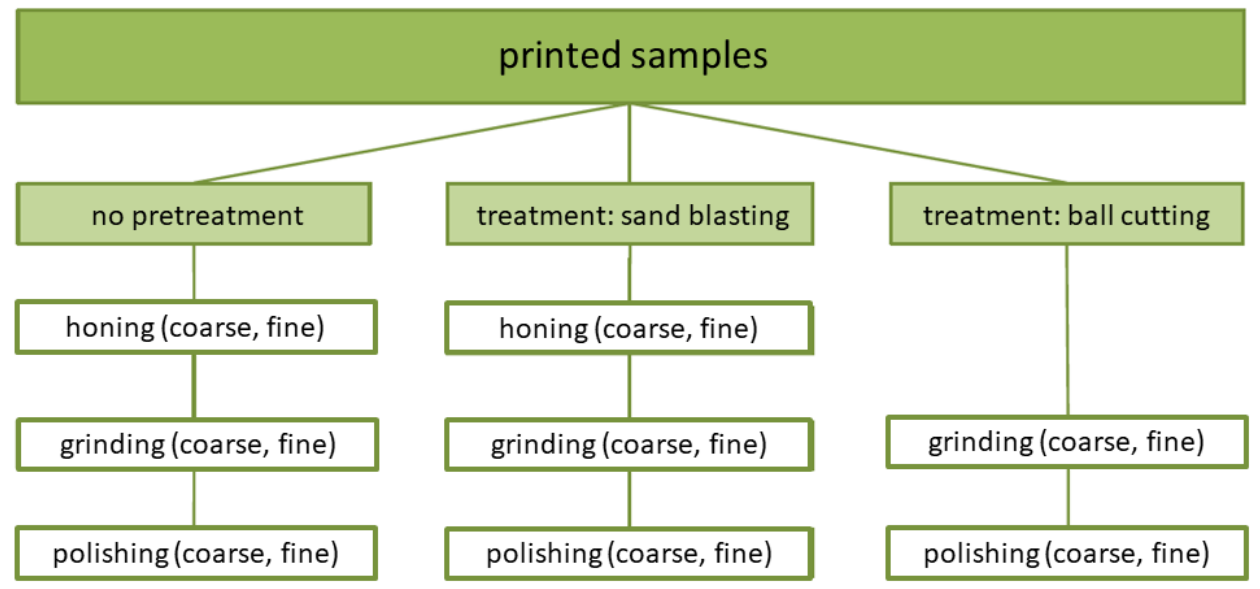

Figure 5: Post-processing procedure for mechanical finishing techniques

The procedure provides three initial situations: The samples are either post-processed without an additional pretreatment or post-processed with a previous sand blasting treatment or milling treatment, in this case ball cutting. This ensures a greater variety of investigated techniques. In case of the ball cutting treatment the honing process has been discarded due to the experience that honing generates rougher surfaces than ball cutting.

Between each post-processing step the samples are measured. The reflectivity of a surface is mainly dependent on two properties: the surface geometry and the chemical composition of the surface. Since the samples are only treated with abrasive techniques, the evaluation of the chemical composition is not further considered. In terms of surface geometry the surface roughness is evaluated. The measured value is $R_{z}$, which has a focus on the minimum and maximum height of the surface. The measurement is conducted with the incident light microscope Keyence VHX-900F microscope and with the laser scanning microscope Keyence VK9700. The resolution in depth of the incident light microscope is expected to be insufficient for at least the polished samples. Therefore two measurement devices are used and compared for the investigation.

A measurement of the reflectivity of the surfaces has been conducted using a goniometer. 


\section{RESULTS}

The measurements of the surface roughness can be seen in figure 6 . The post-processing procedures reduced the initial surface roughness of about $30 \mu \mathrm{m}$ to $3-10 \mu \mathrm{m}$. The pretreatment has an effect on the roughness distribution throughout the surface angles, but the overall value stays the same, which is expected. However, the differences between untreated and pretreated samples are not expected, since surface roughness after the abrasive post-processing should be determined by the last post-processing step that was always polishing.
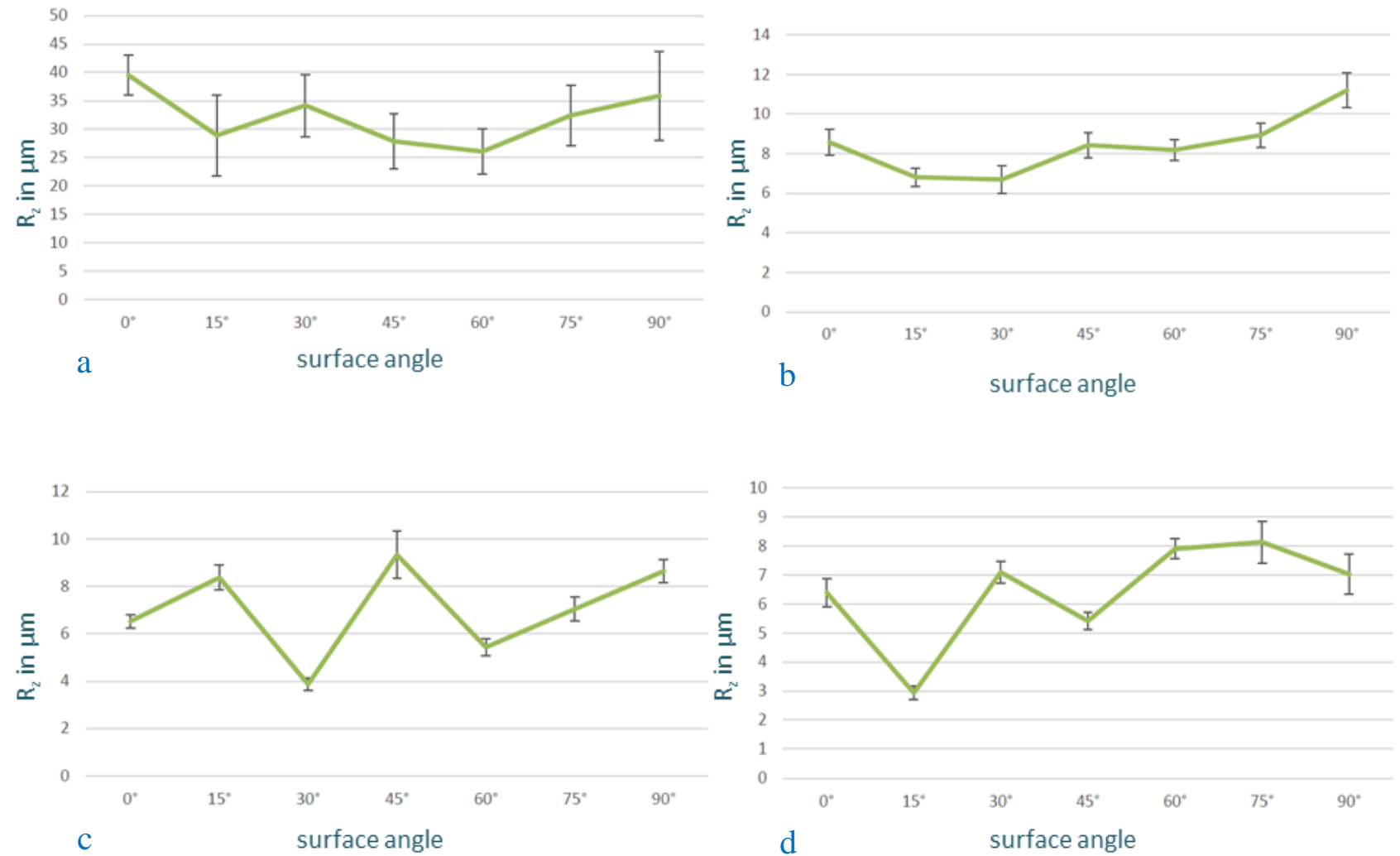

Figure 6: Surface roughness of the different facets of the sample for three initial situations and their standard deviation; a) reference samples as printed, b) honed, grinded and polished without pretreatment, c) honed, grinded and polished with sand blasting as pretreatment and d) grinded and polished with ball cutting as pretreatment

Another effect of the post-process can be seen in figure 7. Here only the pretreatment of ball cutting is investigated. It is shown, that with increasing surface angle the surface roughness also increases. This dependency can directly be seen on the sample that features are glossy surface at $0^{\circ}$. The other surfaces appear less glossy. 

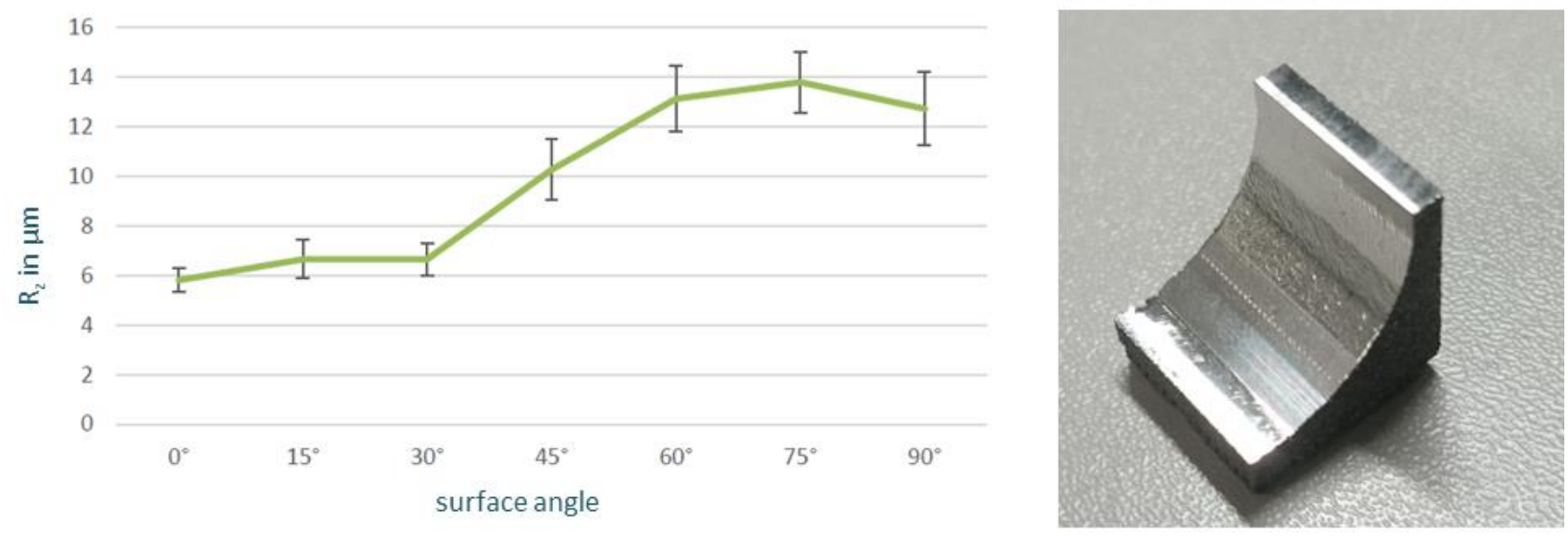

Figure 7: Surface roughness of the facets after ball cutting; surface roughness increases for increased surface angles (left), sample after ball cutting treatment (right)

Figure 8 depicts the achieved reflectivity for each post-processing procedure. It can be seen, that again the pretreatment influences the surface properties. Furthermore the value of the reflectivity is always lower than one percent. Ball cutting combined with grinding and polishing achieves the best value of $0.4 \%$.

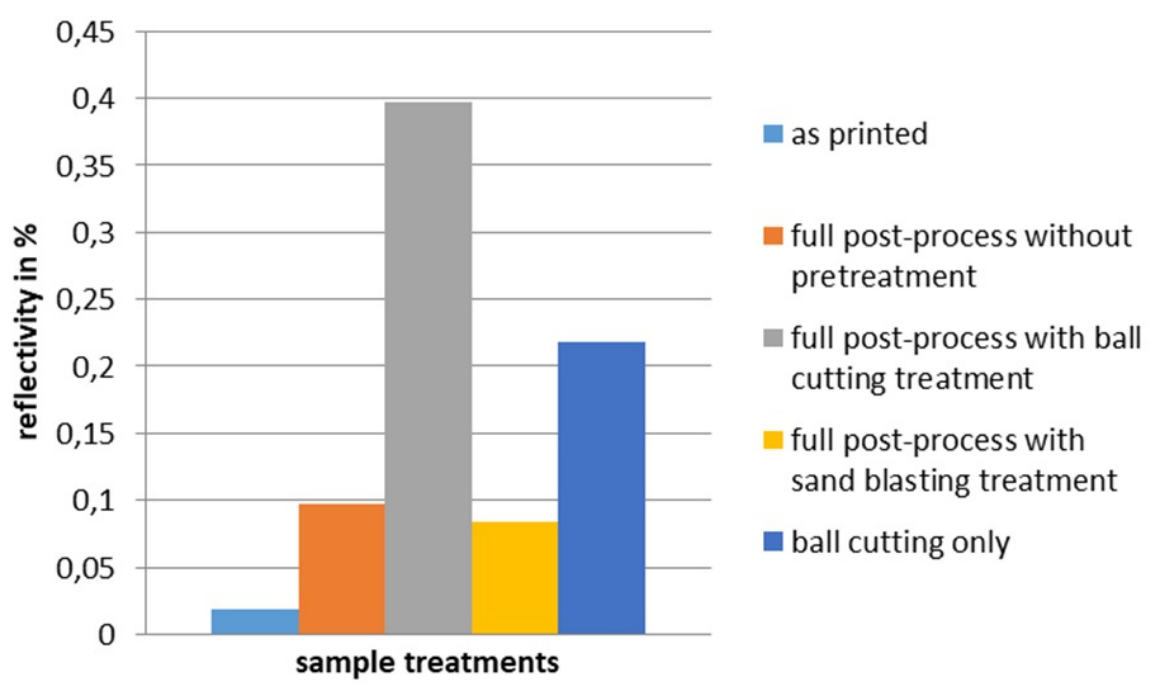

Figure 8: Maximum reflectivity after post-processing with different pretreatments

\section{DISCUSSION}

The results show a partially unexpected behavior. First of all, a pretreatment influences the measured surface roughness, although the last step of polishing should adjust the surface roughness to the same value and the same distribution throughout the surface angles. The second conspicuity is the overall value of $\mathrm{R}_{\mathrm{z}}$. Even after polishing the values stay above $3 \mu \mathrm{m}$ which inflicts heavy losses on the reflectivity. The reason for both effects lies within the manufacturing processes. In contrary to molding or other manufacturing processes, Additive Manufacturing can produce parts with a part density up to $99.8 \%$. However, every hole and imperfection is revealed by abrasive post-processing techniques, regardless how many layers of material have been removed. Therefore abrasive techniques are only effective within a certain range, depending on the initial surface roughness. The differences to roughness values in literature to the measured ones support the statement, since these values have been measured with die casted or molded parts ${ }^{15}$. 
The different roughness values after the ball cutting result from the tool itself. The samples have not been tilted for the finishing of each facet, because the treatment took place on a 3-axis table. Therefore the tool treats the surface of tilted facets with its sides. In order to avoid this effect a 5-axis table should be used rather than a 3-axis table. This step ensures the independence of the surface roughness from the surface angle for post-processing.

Regarding the reflectivity the results are insufficient. The values stay below one percent and not one surface could be used as an imaging optic. However, if the application of the reflector is to scatter light for illumination purpose, some of these techniques can be considered. But nonetheless the reflectivity is limited by the surface, which is limited by the manufacturing process. Summarizing, abrasive post-processing techniques seem inappropriate for the finishing process of reflective optics.

Additive techniques face a similar problem. Depending on the initial surface the added layers can have a better chemical composition for reflectivity, but the geometry of the surface adapts to the initial surface. Of course one could add enough material to fill holes overlay imperfections, but this can result in an ineffective usage of material.

In terms of direct manufacturing the named post-processing techniques are already used for hybrid manufacturing, especially milling or in this case ball cutting. Mechanical polishing is an option for integration into an AM system, but the limitations due to the part itself have to be considered. From the prospective of this paper hybrid manufacturing with mechanical polishing is not favorable.

With respect to the mentioned aspects it is suggested that for post-processing of reflective optics a transformative technique is used. With a rearrangement of the surface the problems of porosity and imperfections due to the AM process can be avoided. Furthermore no additional material is necessary and no additional waste is produced. A technique, which is already used for post-processing transparent optics, is laser polishing where the laser remelts the surface of the reflector. Due to the surface tension the surface smoothens and generates a nearly flat plane. The laser polishing process can be distinguished into to two steps: macro- and micro-polishing. Macro-polishing is used for surfaces with a roughness higher than $40 \mu \mathrm{m}$. In combination with a 5-axis table the surfaces can be treated equally. A tilted surface would deter the laser spot on the surface, hence another treatment influence. Furthermore, the laser polishing technique cannot be used with an SLM machine due to the 5-axis table.

\section{SUMMARY}

This work concentrates on the post-processing techniques for reflective optics. With specifically designed samples different mechanical post-processing techniques have been examined regarding surface roughness and reflectivity. Three post-processing chains have been performed and compared. The surface roughness of ball cut and polished samples was the lowest, resulting in the highest reflectivity. The values for the reflectivity are below one percent and therefore not suited for precision optics. Discussing abrasive techniques the main reason for insufficient surface properties lies within the manufacturing process. Due to the nature of abrasive techniques these issues cannot be overcome easily, thus these techniques are not suitable for implementing in AM systems for the fabrication of reflective optics. A recommendation for hybrid machines has been made using a laser polishing device in a nozzle-based AM machine. Further investigations regarding the hybrid machine will be made.

\section{ACKNOWLEDGEMENTS}

The authors are supported by the Ministry for Science and Culture in Lower Saxony and the program "Tailored Light".

\section{REFERENCES}

1. Horn, T.J. and Harryson, O.L.A., "Overview of current additive manufacturing technologies and selected applications", Science Progress 95(3), 255.282 (2012) 
2. Gao, W. et al., "The status, challenges, and future of additive manufacturing in engineering", Computer-Aided Design 69, 65-89 (2015)

3. Hopkinson, N. and Dickens, P., "Rapid prototyping for direct manufacture." Rapid prototyping journal 7.4, 197-202 (2001)

4. Gibson, I., Rosen, D., and Stucker, B., [Additive manufacturing technologies: 3D printing, rapid prototyping, and direct digital manufacturing], Springer (2014)

5. Chohan, J. S., Chohan, J. S., Singh, R., and Singh, R., "Pre and post processing techniques to improve surface characteristics of FDM parts: a state of art review and future applications", Rapid Prototyping Journal, 23(3), 495513(2017)

6. Łyczkowska, E., Szymczyk, P., Dybała, B., and Chlebus, E., "Chemical polishing of scaffolds made of Ti-6Al-7Nb alloy by additive manufacturing", Archives of Civil and Mechanical Engineering, 14(4), 586-594 (2014)

7. Karunakaran, K. P., Suryakumar, S., Pushpa, V., and Akula, S., "Low cost integration of additive and subtractive processes for hybrid layered manufacturing. Robotics and Computer-Integrated Manufacturing", 26(5), 490-499 (2010)

8. Joshi, P. C., Dehoff, R. R., Duty, C. E., Peter, W. H., Ott, R. D., Love, L. J., and Blue, C. A., "Direct digital additive manufacturing technologies: Path towards hybrid integration", Future of Instrumentation International Workshop (FIIW), pp. 1-4 (2012)

9. Zhu Z., Dhokia V.G., Nassehi A., Newman S.T., "A review of hybrid manufacturing processes - state of the art and future perspectives", International Journal of Computer Integrated Manufacturing, 26-7, 569-615 (2013)

10. Currie, M., and McBride, R., "Rapid manufacture of freeform micro-optics for high power applications", Proc. of SPIE, 8970, 89700T-1 (2014)

11. Sung, Y. L., Jeang, J., Lee, C. H., and Shih, W. C., "Fabricating optical lenses by inkjet printing and heat-assisted in situ curing of polydimethylsiloxane for smartphone microscopy", Journal of biomedical optics, 20(4), 047005-047005 (2015)

12. Lamikiz, A., Sanchez, J. A., de Lacalle, L. L., and Arana, J. L., "Laser polishing of parts built up by selective laser sintering", International Journal of Machine Tools and Manufacture, 47(12), 2040-2050 (2009)

13. Xiong X., Zhang H., Wang G., "Metal direct prototyping by using hybrid plasma deposition and milling", Journal of Materials Processing Technology, 209, 124-130 (2009)

14. Sweatt, W. C., Gill, D. D., Ada, D. P., Vasile, M. J., and Claudet, A. A., "Diamond milling of micro-optics", IEEE Aerospace and Electronic Systems Magazine, 23(1), 13-17 (2008)

15. DeGarmo, E. P., Black, J. T., Kohser, R. A., and Klamecki, B. E., [Materials and process in manufacturing], Prentice Hall (1997) 